\title{
Recording of the Multiplexed Bragg Diffraction Gratings for Waveguides Using Phase Mask
}

\author{
Maria Shishova $\mathbb{D}^{\mathbb{1}}$, Alexander Zherdev, Dmitrii Lushnikov and Sergey Odinokov * \\ Bauman Moscow State Technical University, 2nd Baumanskaya st. 5/1, 105005 Moscow, Russia; \\ mshishova@bmstu.ru (M.S.); zherdev@bmstu.ru (A.Z.); dmlu41@yandex.ru (D.L.) \\ * Correspondence: odinokov@bmstu.ru
}

Received: 21 September 2020; Accepted: 26 October 2020; Published: 27 October 2020

check for updates

\begin{abstract}
The paper describes a stable method for multiplexed recording of the Bragg diffraction gratings for waveguides using a phase mask. Diffraction waveguides in this experiment were made of photo-thermo-refractive glass. Two types of the phase mask are considered: surface and volume. Their comparison is based on diffraction characteristics of manufactured single and multiplexed Bragg gratings. The experimental results showed the advantage of surface phase mask application. To confirm the efficiency of the proposed method, diffraction waveguides was successfully fabricated and integrated in see-through near-eye display.
\end{abstract}

Keywords: diffraction gratings; Bragg gratings; holographic lithography; phase mask; holographic replication; diffraction waveguides; near-eye display

\section{Introduction}

With the development of augmented reality (AR) [1,2] and integrated photonics devices [3], researchers are faced with the task of developing new methods for waveguide holograms recording and creating the diffraction waveguides. In the field of AR displays, diffraction waveguides are the plane-parallel substrates combined with the diffraction gratings that provide substrate-mode propagation of diffracted beams with the help of total internal reflection (TIR). The diffraction grating can be surface or volume one but diffraction angle inside the substrate must satisfy TIR condition. Bragg holographic gratings are commonly used for the grating-based waveguide configurations to increase the optical efficiency of AR systems [4,5]. Corresponding recording is associated with increasing the complexity level since large convergence angles of the recording beams must be provided. An object wave must be inside the substrate during recording. In this paper, a method for multiplex recording of Bragg diffraction waveguide gratings is proposed. Presented waveguides refer to AR planar imaging optics used for radiation coupling into a glass plane-parallel plate.

AR implementation under study includes multiplexed space variant elements and requires holographic materials with good refractive index modulation properties. In the research, the planar waveguides rely on photo-thermo-refractive (PTR) glass as a holographic volume media. This material is unique for the integration of a phase diffraction elements into the waveguide platform [6]. PTR glass is good material for see-through displays because avoids various problems when exposed to environmental perturbations such as temperature, humidity and pressure, and close to optical glass BK7 by the properties [7]. The glass substrate is the holographic material itself so we can record holograms over the entire thickness of the substrate. The good advantage of PTR glass for holography is the absence of shrinkage.

Creation of the waveguide mode multiplexed Bragg gratings in the planar waveguide made from PTR glass is a challenge of the research. We propose a recording method using the phase mask and oblique illumination. This technique is stable and convenient to fabricate high resolution volume 
diffraction gratings [3,8-11] with waveguide mode operation. Presented research is distinguished by oblique illumination of the phase mask with a single recording laser beam to manufacture the slant volume gratings for AR waveguide displays. Conventional phase mask techniques provide recording of the structure with isophase fringes perpendicular to the substrate surface. With such a limitation, it is impossible to create AR waveguides, since their functioning directly depends on the direction of the diffraction structure relative to the substrate surface. The oblique illumination of the proper phase mask allows us to record the slant gratings working in the waveguide mode. The paper considers two types of the phase mask: non-selective surface diffraction gratings and selective volume diffraction gratings. To provide recommendations for their applicability, the comparison is carried out based on diffraction characteristics of manufactured single and multiplexed Bragg gratings. To confirm the efficiency of the proposed method, the diffraction waveguide was successfully fabricated and integrated in see-through AR display.

\section{Principles}

The recording though the phase mask has a number of advantages over the standard interferential recording set up, which implements an amplitude division interferometer. There are several arguments in favor of the phase mask application independent from its type. To provide high frequencies of Bragg gratings, large convergence angles of the recording beams are required [12]. This is easier to implement with a diffraction grating as the phase mask, whereby convergence angles are diffraction angles. Flat uniform wavefront must be created for only one branch. In the described experiment, volume gratings were manufactured in a wide beam of at least $50 \mathrm{~mm}$ in aperture. The entire area of the diffractive waveguide was recorded at the same time. The creation of this size uniform plane wavefront becomes a nontrivial task for UV light. Requirements for a vibration stabilization of the recording set up were significantly reduced. Due to the fact that the sample and the phase mask are combined into a single structure; the mechanical vibrations occur relative to the sandwich from the phase mask and the photosensitive PTR substrate. The interference pattern is not getting shifted during the recording due to the vibrations. It is necessary to stabilize the relative displacement of the mechanical components in the scheme only in one branch, which is beneficial for long-term exposures.

This section describes in detail a general guideline for the recording though the surface and volume phase masks with multiple sequential exposures. Figure 1a illustrates the scheme of the recording set up. The proposed recording method applies the diffraction grating as the phase mask to create a near-field interference pattern in the photo-thermo-refractive substrate. The energy distribution in diffraction orders of the phase mask is responsible for the recording contrast.

Single coherent beam illuminates the phase mask and forms superimposing beams in the diffraction orders, the phase mask gets replicated to the thick-layer holographic material. This method is also called holographic or optical replication $[13,14]$, especially if the recording laser wavelength during the replication is the same as when recording the phase mask. Radiation with the wavelength of $0.325 \mu \mathrm{m}$ (He-Cd laser 1) is expanded and collimated using lenses 2 и 3 as a telescopic Kepler system. A diaphragm 4 cuts the central part of the recording beam with the satisfactory uniformity of the radiation intensity. The sandwich of phase mask and PTR glass substrate 5 is mounted on a motorized angular positioner 6 that controls an angle of incidence $\alpha$. Coherent plane wave falls onto the one-dimensional grating (phase mask) first, a plane of incidence is normal to the grooves of the phase mask. The exposing area in the recording beam is controlled by the displacement in the $x y$ plane. The entire recording procedure with the necessary angular and planar positions is carried out using programmed control of the actuators in the recording setup. The exposure dosage is provided by an electromechanical shutter 7 . 


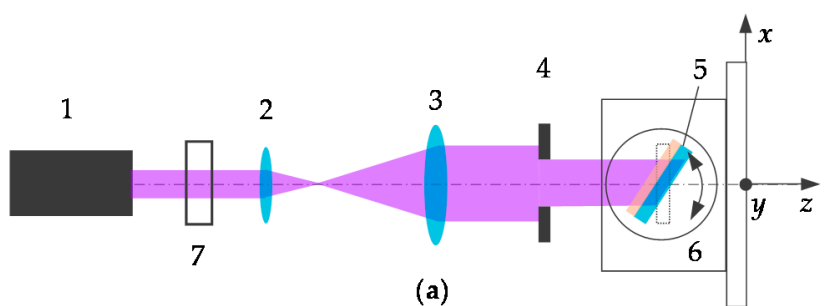

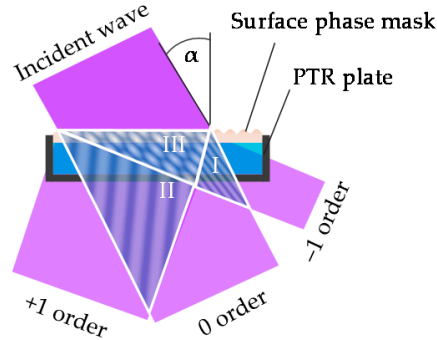

(b)

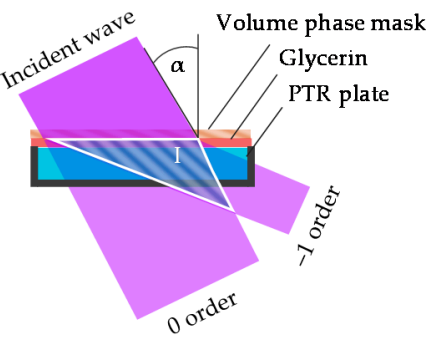

(c)

Figure 1. (a) Scheme of the recording set up: 1-He-Cd laser, 2, 3-lenses, 4-diaphragm, 5-mask/waveguide sandwich in conical exposition, 6-angular positioner, 7-shutter. Near-field area formed by single exposure of (b) surface phase mask and (c) Bragg phase mask.

Figure $1 b, c$ represents the near-field recording areas formed after surface and volume gratings, respectively. The phase mask must be directly applied to the waveguide or must be adjacent through an immersion liquid in order to correctly implement the conditions for large convergence angles. Diffraction on the non-selective surface grating (Figure 1b) as the phase mask results in three beams propagating inside the PTR glass substrate: transmitted beam (0th diffraction order) and two diffracted beams (+1st and -1 st orders). Therefore, three interference areas are formed in te PTR glass substrate: the area $I$ corresponds to interference of +1 st and 0 th diffraction orders, the area $I I$ with interference of 0 th and -1 st diffraction orders and the area III with interference of all three +1 st, 0 th, and -1 st diffraction orders. Figure $1 \mathrm{~b}$ illustrates a geometrical representation. In practice, the area III occupies most of the recording volume due to relatively small waveguide thickness $(2 \mathrm{~mm})$, in comparison with the aperture of the recorded beam $(40 \mathrm{~mm})$. The interference pattern of the area III fills the full thickness of the holographic material. The size of the recording beam is chosen so that its aperture is sufficient to fill the entire required area of the final diffraction element. Still non-uniformity can impact the diffraction efficiency. The following experiments represents the resulting performance that can be achieved. Diffraction on the selective volume grating (Figure 1c) as the phase mask results in two waves that are propagating inside the PTR glass substrate: transmitted wave (0th order) and diffracted wave ( -1 st order). The operating periodicity of the diffraction waveguide is formed by the interference of 0 th and -1 st diffraction order, which is included in the areas I and III.

The waveguide couplers for AR displays consist of several multiplexed volume Bragg gratings. Figure 1 represents a single exposure step. The experimental task of multiplexing procedure is to use effectively the refractive index modulation, which is responsible for the entire dynamic range.

\section{Experiment Results}

\subsection{Recording and Development Conditions}

The holographic replication set up is vibration resistant as aforementioned. Recording took place under the vibration conditions shown in Figure 2a. The graph shows the deviations controlled by microinterferometer displacement sensor during $1.25 \mathrm{~h}$. A single exposure time with multiplex recording is much less than the tracking time presented (it takes two minutes). Linear displacements significantly exceed the period of the recorded Bragg diffraction gratings during the recording described. 


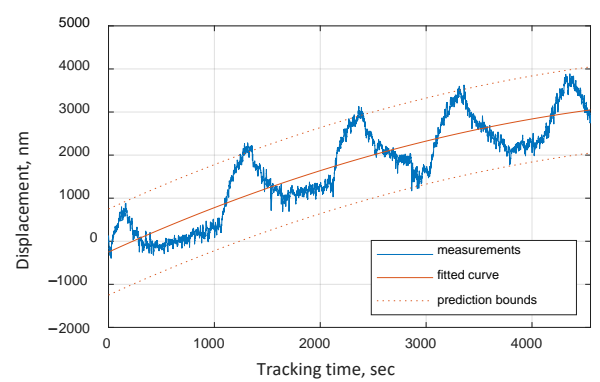

(a)

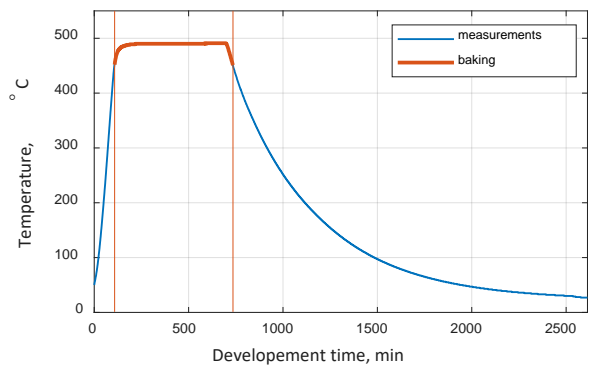

(b)

Figure 2. Recording and development conditions: (a) Vibration/time conditions during recording, (b) temperature/time dependence during baking.

The post-exposure development for PTR glass consists of the high-temperature treatment. The baking took $10 \mathrm{~h}$ at the temperature $\approx 500{ }^{\circ} \mathrm{C}$, the temperature graph is shown in Figure $2 \mathrm{~b}$. High temperature treatment is the developing procedure for PTR glass when the refractive index modulation gets fixed [7].

\subsection{Measurement of Diffraction Efficiency of Waveguide Gratings}

This section considers the recording experiments comparing two types of the phase masks. The diffraction efficiency is represented as a function of the exposure dose. The phase masks were designed to create a green spectrum waveguide mode for incidence angles from $-15^{\circ}$ to $15^{\circ}$ to create the substrate TIR-guided beam. The recording angles for multiplexing were varied in the range from $0^{\circ}$ to $30^{\circ}$. Figure 3 shows the exposure curves for the recording through the surface and Bragg phase masks, respectively. He-Cd laser Kimmon IK3501R-G with power $50 \mathrm{~mW}$ and wavelength $325 \mathrm{~nm}$ was used for recording. The exposure values shown in the plots refer to the radiation illuminating the phase mask. After the beam expansion, the laser power in the beam arriving to the phase mask was about $2 \mathrm{~mW}$.

Blue exposition curves on the left y-axis represent the dependence of the diffraction efficiency on the exposure dose. The exposure is the power density needed to provide effective photosensitive response of the holographic medium. On the right y-axis, a similar dependence for the transmittance is shown in red. A feature of the waveguide diffraction gratings control is the difficulty of direct measurements of the diffraction efficiency, due to the fact that the -1st diffraction order is trapped inside the substrate. Therefore, the study is based on an indirect control by an extinction measuring the power of the transmitted beam. It is shown that for both types of phase masks, effective exposure power density is $1-2 \mathrm{~J} / \mathrm{cm}^{2}$.

The waveguide couplers for AR displays consist of several multiplexed volume Bragg gratings. The angular step for multiplexing is determined by the angular selectivity of recorded Bragg grating and spectral range of the reconstruction light-emitting diode. Figure 1 represents a single exposure step. The experimental task of multiplexing procedure is to use effectively the refractive index modulation $\Delta \mathrm{n}$, which is responsible for the entire dynamic range. It is distributed to $\mathrm{N}$-fold multiplexing, resulting in refractive index modulations $n_{1}, n_{2}, \ldots, n_{N}$. The diffraction efficiency of individual grating in the multiplex decreases approximately by the square root of $\mathrm{N}$ times [15].

Figure 4 shows the results of indirect measurement for multiplex Bragg gratings recorded and represents the bleaching impact. The bleaching was provided by pulsed $250 \mathrm{fs}$ laser, with wavelength $515 \mathrm{~nm}$, using direct beam scanning at the rate of $0.02 \mathrm{~mm} / \mathrm{s}$. Due to photodestruction of silver nanoparticles inside the PTR glass, a decrease in scattering and absorption occurred, increasing the transparency. The diffraction efficiency gets higher for a couple percent and the transmission for about $10 \%$. Although Bragg phase masks should provide better contrast while recording, any positive 
multiplexing results were not achieved for this type mask. A further experiment to increase the number of multiplexes was carried out only for the surface phase mask.

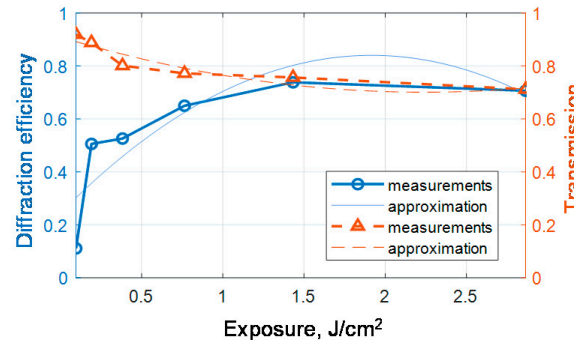

(a)

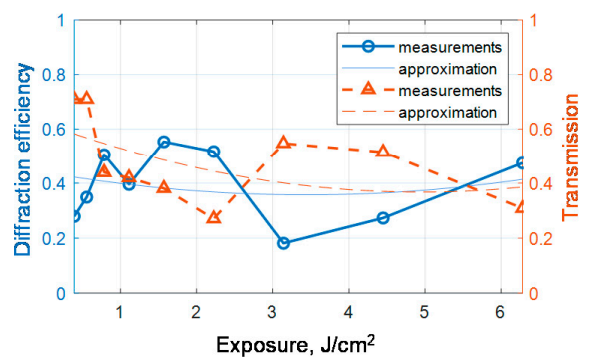

(d)

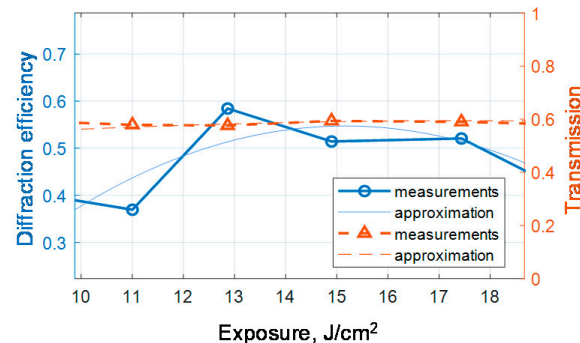

(b)

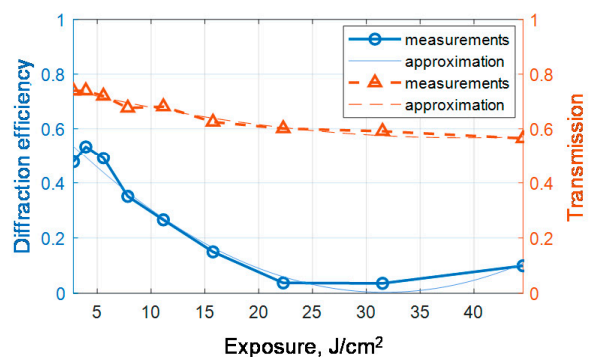

(e)

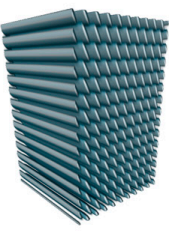

(c)

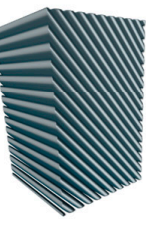

(f)

Figure 3. Exposure curves for grating recorded with the surface phase mask for (a) small exposures and (b) large exposure doses and with Bragg phase mask for (d) small exposure doses and (e) wide range of exposure doses. 3D-simulation single phase structure in photo-thermo-refractive glass recoded using (c) surface phase mask and using (f) Bragg phase mask.

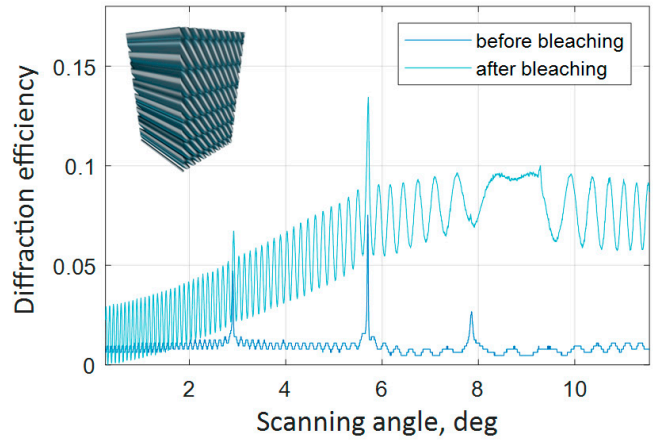

(a)

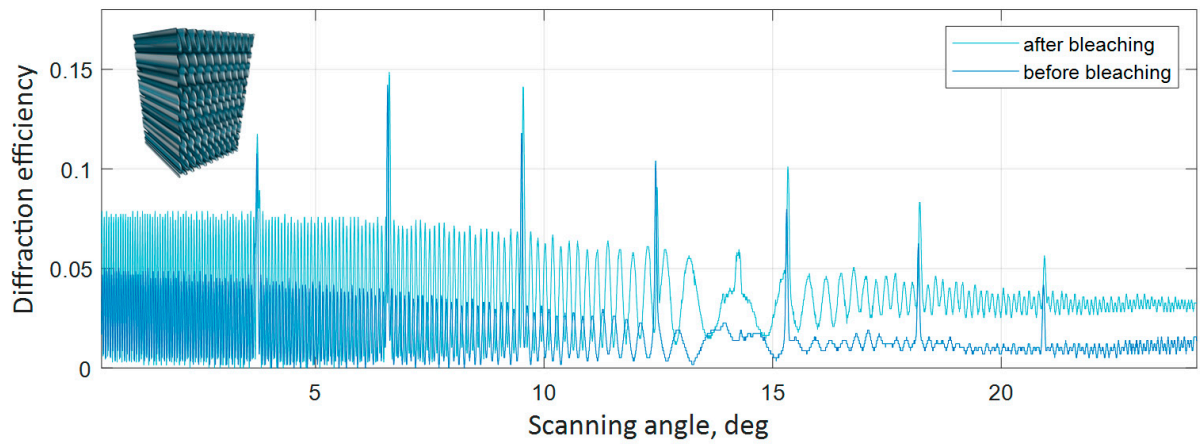

(b)

Figure 4. Measurements of the diffraction efficiency via exposition dose of multiplexed grating: (a) Recorded by Bragg phase mask with five exposures; and (b) relief phase mask with seven exposures. 
Figure 5 a shows the result of multiplexing using surface phase mask. It is shown how increasing of multiplex number affects the diffraction efficiency while maintaining the total exposure. The diffraction efficiency of a single grating is shown in blue. Red and yellow shows the distribution of diffraction efficiency for five and ten multiplex diffraction gratings. Experiments have shown that for the $2 \mathrm{~mm}$ waveguide used, it was possible to achieve 10-20\% diffraction efficiency in each of the 10 multiplexes. On the basis of described multiplex diffraction elements, it is possible to create an augmented reality waveguide guide for image transmission [6]. Figure $5 b$ illustrates one substrate guided beam from the green laser light propagating by TIR inside the manufactured planar waveguide. Multiplexed volume holographic gratings are used as the optical couplers. Each multiplex provides its own coupling angle responsible for the sub-angular field-of-view in the augmented reality system. The in-coupler is employed to modulate the propagation directions and diffraction efficiencies of the collimated beams from the image projector. According to the selectivity contours of the in-coupler, the light beams are inputting into the waveguide and then propagates along the corresponding direction. The out-coupler works in the opposite way. It is located near eye and outputs the light beams from the substrate in the same angular direction as the incident beams were. Demonstrated in the paper, in- and out-couplers are identical and recorded with the same exposures. This display includes seven multiplexed Bragg gratings, recorded with the help of the surface phase mask. In the case under research, for the in-coupler at negative angles at Figure 5 a of incidence in the air, multiplexed gratings are created to operate in the transmission mode, and for positive angles of incidence in the air-in the reflective mode. The symmetrical situation for the out-coupler, half of the angular field is provided by reflection diffraction modes, and half by transmission diffraction modes. Figure $5 c$ shows the same plane-parallel plate implemented in see-through display with outputted green image from compact projector with wide green spectrum.

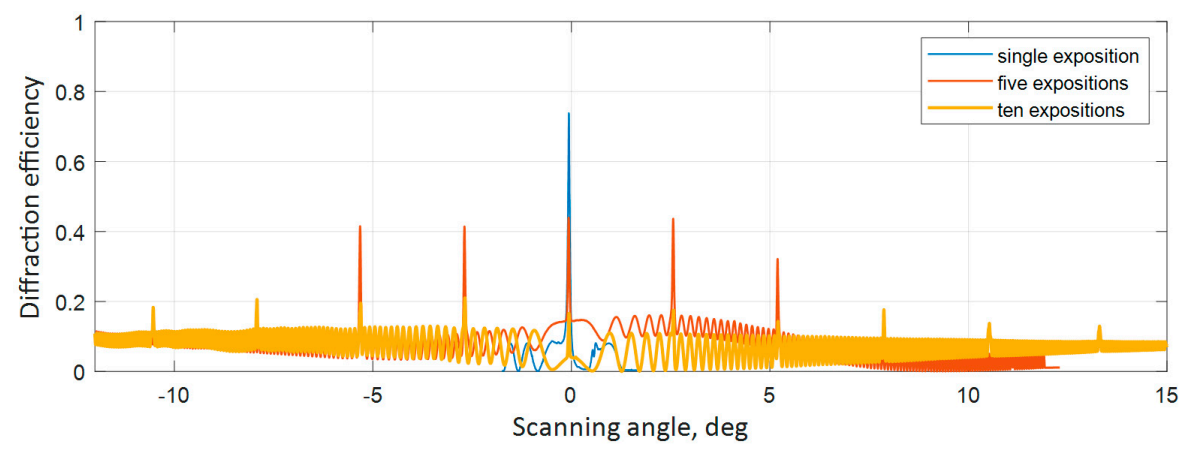

(a)

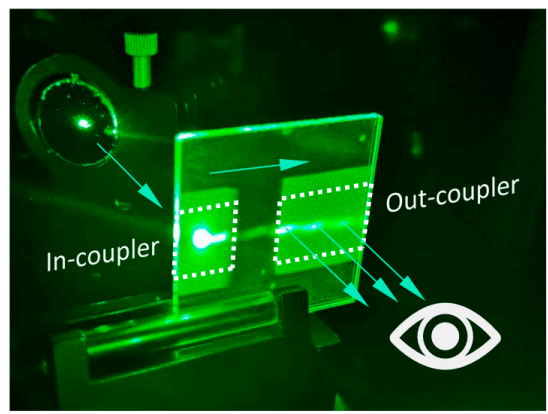

(b)

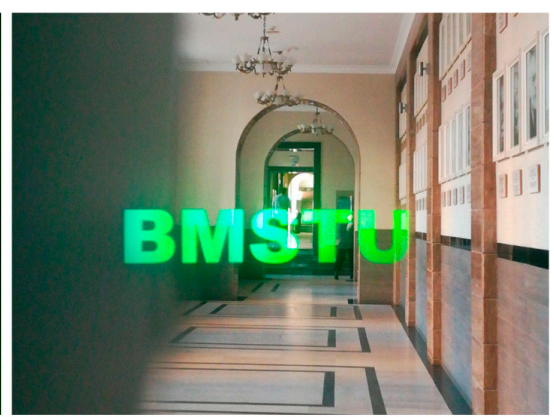

(c)

Figure 5. (a) Diffraction efficiency via scanning angle recorded with relief phase mask with different multiplexing conditions. (b) Illustration of diffraction waveguide work with one inputted substrate-mode beam inside the photo-thermo-refractive plane-parallel plate. (c) The outputted image on see-through display based on diffraction waveguide with seven multiplexes. 


\section{Discussion}

The multiplexed recording through the phase mask in PTR glasses was carried out in the research. In the course of the study, a comparison was made between two types of phase masks: the surface non-selective phase mask and the volume selective phase mask. The design of the phase mask itself is not the item of the research presented. It is based on a diffraction equation and starts with the periods and slant angles of the Bragg grating that needs to be achieved [6].

Multi-exposure approach is analyzed to balance the angular bandwidth and diffraction efficiency of the resulting volume diffraction elements. The multiplexing helps to expand the effective angular field of view in near-eye displays. The structural parameters for each multiplexed grating are optimized to the propagation direction. The space multiplexing varies the slant angles of the volume Bragg grating in the diffraction couplers. The presented procedure is more feasible for the application of the surface phase mask. Rearrangement of the interference recording set up for a wide range of multiplexing angles is complicated. However, having recorded the phase mask once and performing the replication procedure, we rotate only one component in the recording beam. When recorded in the photoresist, the surface phase mask can be applied directly to the PTR glass plate. PTR glass is not photosensitive to radiation, which is actinic for the photoresist. In the other case, the selective phase mask is no longer applied for angular multiplexing; thus, it is necessary to replace the phase mask for each following multiplex and, accordingly, make as many masks as necessary for multiplexing. Additionally, for each exposition step we need to use immersion liquid. The selective phase masks presented in this research were made from dichromated gelatin.

It was expected that the application of Bragg grating as the phase mask would lead to more efficient refractive index modulation of the medium, whereby it was possible to achieve equalization of the diffraction efficiency in two orders. Nevertheless, the difficulties associated with the immersion liquid application and docking of the various selective masks complicate the process and have a destructive effect. During the experiment, it was not possible to achieve positive results in multiplexing using the selective phase masks due to the increase in the technological complexity.

The surface phase mask provides quality multiplexed recording despite the inequality of diffraction efficiency in the orders. This type masks shows the same results in exposure dosages as Bragg phase masks, but their application is more convenient due to the free control of the recording beam angle due to the non-selectivity. The holographic replication of the surface phase mask is the suitable recording method for multiplexed slanted volume diffraction gratings.

Presented method reduces the period of the recorded optical phase structures by 1.5 times in comparison with the period of the phase mask and shows the high level of repeatability. This recording technique is accompanied with no need in the strong vibration isolation because of the absence of the interferometric branches in the optical scheme. The presented method can be applied to Bragg grating recording in optical fibers. PTR glass helps to integrate thick holographic grating inside the planar dielectric substrate. The recorded multiplexed volume gratings can be tailored for the coupling functions for AR platform. The presented example of the waveguide for the near-eye display demonstrates the operability of multiplex diffraction elements recorded using the technique under study.

Author Contributions: Conceptualization, supervision, project administration, and funding acquisition S.O.; methodology S.O. and D.L.; resources, phase mask recording D.L.; formal analysis, multiplexed Bragg grating recording, software, validation and data curation M.S. and A.Z.; investigation M.S., A.Z. and D.L.; visualization, writing-original draft preparation and writing_-review \& editing M.S. All authors have read and agreed to the published version of the manuscript.

Funding: This research was funded by Ministry of Science and Higher Education of the Russian Federation, grant number 0705-2020-0041.

Acknowledgments: The authors acknowledge the support by Vladimir Markin for phase masks calculations, Dmitrii Kuzmin, Pavel Khanevich and Ekaterina Drozdova for their help in technical issues. The authors thank Nikolay Nikonorov and Sergey Ivanov for consultations on photo-thermo-refractive glass. 
Conflicts of Interest: The authors declare no conflict of interest.

\section{References}

1. Zhang, Y.; Fang, F. Development of planar diffractive waveguides in optical see-through head-mounted displays. Precis. Eng. 2019, 60, 482-496. [CrossRef]

2. Lee, Y.-H.; Zhan, T.; Wu, S.-T.; Yun-Han, L.; Tao, Z.; Shin-Tson, W. Prospects and challenges in augmented reality displays. Virtual Real. Intell. Hardw. 2019, 1, 10-20. [CrossRef]

3. Hsu, F.-H.; Han, C.-Y.; Chen, K.-H.; Hsu, K.-Y.; Chen, J.-H. Prism-hologram-prism sandwiched recording method for polarization-selective substrate-mode volume holograms with a large diffraction angle. Opt. Express 2018, 26, 20534-20543. [CrossRef] [PubMed]

4. Yu, C.; Peng, Y.; Zhao, Q.; Li, H.; Liu, X. Highly efficient waveguide display with space-variant volume holographic gratings. Appl. Opt. 2017, 56, 9390. [CrossRef] [PubMed]

5. Shen, Z.; Zhang, Y.; Liu, A.; Weng, Y.; Li, X. Volume holographic waveguide display with large field of view using a Au-NPs dispersed acrylate-based photopolymer. Opt. Mater. Express 2020, 10, 312-322. [CrossRef]

6. Odinokov, S.; Shishova, M.; Markin, V.; Lushnikov, D.; Zherdev, A.; Solomashenko, A.; Kuzmin, D.; Nikonorov, N.; Ivanov, S. Augmented reality display based on photo-thermo-refractive glass planar waveguide. Opt. Express 2020, 28, 17581. [CrossRef] [PubMed]

7. Nikonorov, N.; Ivanov, S.; Dubrovin, V.; Ignatiev, A. New photo-thermo-refractive glasses for holographic optical elements: Properties and applications. In Holographic Materials and Optical Systems; InTech: London, UK, 2017; pp. 435-461. [CrossRef]

8. Park, T.-H.; Kim, S.-M.; Oh, M.-C. Polymer-waveguide Bragg-grating devices fabricated using phase-mask lithography. Curr. Opt. Photonics 2019, 3, 401-407.

9. Weichelt, T.; Vogler, U.; Stuerzebecher, L.; Voelkel, R.; Zeitner, U.D. Resolution enhancement for advanced mask aligner lithography using phase-shifting photomasks. Opt. Express 2014, 22, 16310-16321. [CrossRef] [PubMed]

10. Smith, B.W. Design and analysis of a compact EUV interferometric lithography system. J. Micro/Nanolith. MEMS MOEMS 2009, 8, 021207. [CrossRef]

11. Shaoxin, S.; Xuechang, R.; Shou, L.; Zhilin, Y.; YuanYing, Z. Fabrication of large-scale photonic phased array using a holographic lithography system. Opt. Eng. 2013, 52, 095103. [CrossRef]

12. Bobrinev, V.I.; Kovalev, M.S.; Odinokov, S.B.; Sagatelyan, G.R. Investigation of the Properties of a Beam Reconstructed from Volume Holographic Optical Elements Used in Optical Observation Devices. Russ. Phys. J. 2016, 58, 1457-1466. [CrossRef]

13. Vanin, V.A. Hologram copying (Review). Sov. J. Quantum Electron. 1978, 8, 809-818.

14. Odinokov, S.B.; Zherdev, A.Y.; Shishova, M.V.; Solomashenko, A.B.; Lushnikov, D.S.; Markin, V.V. Bragg grating inscription in photosensitive materials by an optical replication process. Quantum Electron. 2020, 50, 653-657. [CrossRef]

15. Zhai, Q.; Tao, S.; Zhang, T.; Song, X.; Wang, D. Investigation on mechanism of multiple holographic recording with uniform diffraction efficiency in photopolymers. Opt. Express 2009, 17, 10871-10880. [CrossRef]

Publisher's Note: MDPI stays neutral with regard to jurisdictional claims in published maps and institutional affiliations.

(C) 2020 by the authors. Licensee MDPI, Basel, Switzerland. This article is an open access article distributed under the terms and conditions of the Creative Commons Attribution (CC BY) license (http://creativecommons.org/licenses/by/4.0/). 